\title{
RELATIONSHIP BETWEEN RUNNING ECONOMY AND MECHANICAL CHARACTERISTICS OF TRICEPS SURAE ASSESSED WITH TENSIOMYOGRAPHY: A PILOT STUDY
}

\author{
Diego Moreno-Pérez ${ }^{1}$, Álvaro López-Samanes², Aitor Centeno ${ }^{3}$, \\ Jonathan Esteve-Lanao ${ }^{4}$, and Ignacio Diez-Vega ${ }^{5}$ \\ ${ }^{1}$ Departament of Education, Research and Evaluation Methods, \\ Comillas Pontifical University, Madrid, Spain \\ ${ }^{2}$ Exercise Physiology Group, School of Physiotherapy, Faculty of Health Sciences, \\ Universidad Francisco de Vitoria, Madrid, Spain \\ ${ }^{3}$ Faculty of Sport Sciences, Universidad Europea de Madrid, Madrid, Spain \\ ${ }^{4}$ AIYM Training System, Mérida, Mexico \\ ${ }^{5}$ Departamento de Enfermería y Fisioterapia, Facultad de Ciencias de la Salud, \\ Universidad de León, Spain
}

Original scientific article

DOI $10.26582 / \mathrm{k} \cdot 52.2 .12$

\begin{abstract}
:
Tensiomyography (TMG) is a non-invasive technique commonly used for evaluating muscle properties in highly trained athletes. The aim of our study was to evaluate the mechanical characteristics of $m$. triceps surae in competitive runners through TMG measurement and analyze if there was a relationship with running economy (RE). Nine male runners completed the study (mean \pm SD: age $40.4 \pm 9.0$ years, body height $176.2 \pm 4.9 \mathrm{~cm}$, body mass $70.7 \pm 9.4 \mathrm{~kg}, 10-\mathrm{km}$ time $\left.39.8 \pm 5.9 \mathrm{~min}, \mathrm{VO}_{2 \text { peak }} 56.9 \pm 6.5 \mathrm{~mL} \mathrm{~kg}^{-1} \mathrm{~min}^{-1}\right)$. Each subject visited the lab on two occasions with $72 \mathrm{~h}$ of rest between the trials. On the first day, an incremental test was performed to determine their ventilatory thresholds and peak oxygen consumption. On the second day, RE was evaluated on a treadmill at the velocity of their first ventilatory threshold (VT1), and mechanical characteristics of the soleus and gastrocnemius muscles were analyzed with TMG. Significant differences were found between the economic and non-economic runners in $m$. soleus in delayed time (Td), contraction time (Tc), and maximal radial displacement of the muscle belly (Dm). Also, significant differences were found in contraction time (Tc) in medium calf $(\mathrm{MC})$ and in half relaxation time (Tr) in lateral twin (LT). The main finding of our study was that the runners with better RE showed greater stiffness in the triceps surae muscles, an aspect that seems to be associated with better performance in athlete runners.
\end{abstract}

Key words: running economy (RE), tensiomyography (TMG), $m$. triceps surae, $m$. gastrocnemius, $m$. soleus

\section{Introduction}

Athletic performance in endurance sports requires the interaction of many factors, some of which are trainable (i.e. physiology, biomechanics, and psychology) and others are teachable (i.e. tactics) (Spurrs, Murphy, \& Watsford, 2003). With regard to training, the scientific literature refers to both physiological and biomechanical factors in an integral manner. These factors are defined as maximum oxygen uptake (VO2max), anaerobic threshold (AT), running economy (RE), and anaerobic capacity/power for short-term efforts (Lucia, et al., 2006; Saunders, Pyne, Telford \& Hawley, 2004).
Some of these factors have a higher degree of trainability and their development depends on genetic aspects as well as age (Smith, 2003). Currently, RE is one of these factors that has attracted the attention of researchers because of its importance for endurance performance (Lucia, et al., 2006; Nummela, Keränen, \& Mikkelsson, 2007; Sawyer, et al., 2010). RE is the steady-state oxygen uptake (VO2) required at a given constant, submaximal velocity (Nummela, et al., 2007). Also, RE is considered to be the best predictor of running performance in well-trained athletes (Saunders, et al., 2004; Sawyer, et al., 2010), especially when effort duration is greater due to the role that it plays in multiple 
key aspects such as thermoregulation, neuromuscular fatigue, and the amount of energy stored that could limit performance (Noakes, 1991).

Traditionally, many factors have been shown to influence RE, such as resistance and endurance training (Balsalobre-Fernández, Santos-Concejero, \& Grivas, 2016; Saunders, et al., 2004), altitude exposure (Burtscher, Gatterer, Faulhaber, Gerstgrasser, \& Schenk, 2010), muscle fiber distribution (Bosco, et al., 1987), environmental factors (Green, et al., 2000), and anthropometric measurements (Støren, Helgerud, \& Hoff, 2011). RE also depends on biomechanical factors such as kinematic and kinetic variables (Saunders, et al., 2004) to a great extent. Therefore, scientific literature has emphasized shorter ground contact times, lower stride frequencies, longer swing times, greater stride angles, and longer strides (Santos-Concejero, et al., 2013, 2014).

In relation to $\mathrm{RE}$, the interaction between muscular stiffness and running biomechanics have increased in the last few years. On one hand, some authors consider this to be an anthropometric factor (Saunders, et al., 2004), while others affirm that it is a biomechanical factor due to its influence on the movement pattern while running (Spurrs, et al., 2003). Long-distance runners, who are able to stiffen the musculotendinous junction of the lower train in the support phase of the running action, have mechanical advantages that permit a lower energy expenditure (Novachek, 1998). Thus, it is known that there is a positive relationship between leg stiffness and RE (Dumke, Pfaffenroth, McBride, \& McCauley, 2010; Fletcher, Esau, \& MacIntosh, 2010; Spurrs, et al., 2003).

During the running action, the central nervous system coordinates the actions of muscles in the supporting leg, tendons, and ligaments so that the whole group behaves similarly to a spring with a mass (Dumke, et al., 2010; Millet, Jaouen, Borrani, \& Candau, 2002). The muscles are commonly defined such as "tensors" and the tendons as "springs". Leg stiffness is the ratio between the maximum force applied to the spring (i.e. the maximum force applied to the ground) and maximum compression of the leg (i.e. the lowering of the center of gravity or the hip's change in vertical height) (Morin, Samozino, Zameziati, \& Belli, 2007). The energy stored in these springs (i.e. muscles and tendons) could limit muscle activation and energy expenditure. Saunders et al. (2004) found that without the return of stored elastic energy, especially from the Achilles tendon and plantar fascia of the foot, the $\mathrm{VO} 2$ would increase $30-40 \%$ when running. Several studies have suggested stiffness as a likely mechanism of particular importance for running (Millet, et all., 2002; Saunders, et al., 2004). To date, stiffness has been measured through a force platform (Dumke, et al., 2010; Millet, et al., 2002;
Spurrs, et al., 2003), mathematical models based on kinetic and kinematic patterns (Morin, Dalleau, Kyröläinen, Jeannin, \& Belli, 2005) using ultrasound (Arampatzis, et al., 2006), dynamometers (Fletcher, et al., 2010; Kubo, Kanehisa, Ito, \& Fukunaga, 2001), or optoelectronic devices (SantosConcejero, et al., 2014).

Tensiomyography (TMG) is a tool used to evaluate the mechanical characteristics of superficial muscles (Valencic \& Knez, 1997, Valencic, Knez \& Simunič, 2001). Initially, it was used for diagnosing neuromuscular pathologies (Valencic, 1990). At present, it has applications in the healthcare field (Maeda, et al., 2018) and in the sports-training field (Zubac \& Šimunič, 2017). TMG has a similarity with other mechanomyographic methods (Šimunič, 2019). More advantageously, TMG is a technical measurement that presents with fewer difficulties with respect to phonomyography and vibromyography, which present low signal-to-noise ratios, high variability, complex measuring setups, expensive hardware, and necessary postprocessing of the signals (Orizio, 2002). TMG is also a tool that can measure the mechanical characteristics of the radial displacement of the muscle belly's transverse fibers (maximal radial displacement, Dm) (Valenčič \& Knez, 1997).

During submaximal race efforts, soleus (SO) and gastrocnemius medialis (GM) were the two main contributors to propulsion and support (Hamner, Seth, \& Delp, 2010). Ball and Scurr (2011) showed a greater contribution during the race phase in the GM and SO in comparison to the gastrocnemius lateralis (GL) (Ball \& Scurr, 2011). On the other hand, as one approaches maximum velocities, the contributions of the biceps femoris (FB) and rectus femoris (FR) increase (Albertus-Kajee, Tucker, Derman, Lamberts, \& Lambert, 2011). Considering that the TMG is a tool that allows to evaluate the mechanical characteristics of the muscle (which can also affect the RE), the objective of our study was to analyze if there was a relationship between the RE and the mechanical characteristics of the most relevant musculature during running races. Our hypothesis was that mechanical characteristics of the triceps surae muscles of the most efficient runners would differ from those of the least efficient runners.

\section{Methods}

\section{Participants}

Nine experienced endurance male athletes completed the study (mean \pm SD: age $40.4 \pm 9.0$ years, body height: $176.2 \pm 4.9 \mathrm{~cm}$, body mass: $70.7 \pm$ $9.4 \mathrm{~kg}, 10-\mathrm{km}$ time: $39.8 \pm 5.9 \mathrm{~min}, \mathrm{VO}_{2 \text { peak }}: 56.9 \pm 6.5$ $\left.\mathrm{mL} \cdot \mathrm{kg}^{-1} \mathrm{~min}^{-1}\right)$. The initial sample was 13 subjects. We divided the sample of the study by calculating the tertiles according to the RE variable and delin- 
eating three groups: (a) economic $(n=5)(b)$ intermediate $(n=4)(c)$ non-economic $(n=4)$. It is worth mentioning that the four subjects of the intermediate group were discarded, leaving only the subjects of economic and non-economic groups to be evaluated. Physical characteristics of the subjects are presented in Table 1. All of the participants volunteered and gave written informed consent to participate in the study, which had been approved by the European University Ethics Committee.

\section{Experimental protocol}

Tests were conducted in the Exercise Physiology Laboratory of the European University of Madrid (i.e. $600 \mathrm{~m}$ altitude). All evaluations were carried out at the same time of day (i.e. afternoon, between 16:00 and 21:00 p.m.) under similar environmental conditions (i.e. $20-25^{\circ} \mathrm{C}$ temperature, $60-65 \%$ relative humidity) to avoid the effects associated with circadian rhythms in sports performance (LopezSamanes, et al., 2017). The first-day evaluation was carried out after a period of 72 hours without vigorous training. Participants were advised not to ingest any stimulant beverages or sports drinks before the test. After a 20-minute standardized warm-up of continuous running on a treadmill (Technogym Run Race 1400 HC, Gambettola, Italy) at $60 \%$ of each participant's maximum heart rate (HRmax) and a block of dynamic warm-up exercises (Ayala, et al., 2016), subjects performed a maximum oxygen consumption test $\left(\mathrm{VO}_{2 \max }\right)$ with a gas analyzer (VO2000, Medical Graphics Corporation, St. Paul, MN, USA). The following variables were measured: oxygen consumption $\left(\mathrm{VO}_{2}\right)$, pulmonary ventilation (VE), ventilatory equivalents for oxygen $\left(\mathrm{VE} \cdot \mathrm{VO}_{2}{ }^{-1}\right)$, and carbon dioxide $\left(\mathrm{VE} \cdot \mathrm{CO}_{2}{ }^{-1}\right)$, and end-tidal partial pressure of oxygen $\left(\mathrm{P}_{\mathrm{ET}} \mathrm{O}_{2}\right)$ and carbon dioxide $\left(\mathrm{P}_{\mathrm{ET}} \mathrm{CO}_{2}\right)$. The all-out protocol was executed with a constant treadmill inclination of $1 \%$ (Jones \& Doust, 1996) at a speed of $10 \mathrm{~km} \cdot \mathrm{h}^{-1}$, with increments of $0.3 \mathrm{~km} \cdot \mathrm{h}^{-1}$ every 30 seconds until volitional exhaustion (Esteve-Lanao, Foster, Seiler, \& Lucia, 2007). Peak Oxygen Consumption $\left(\mathrm{V}^{\circ} \mathrm{O}_{2 \text { peak }}\right)$ was recorded as the highest $\mathrm{VO}_{2}$ value obtained for any continuous $30 \mathrm{~s}$ period during the tests. The VT1 was determined by an increase in both $\mathrm{VE} \cdot \mathrm{VO}_{2}{ }^{-1}$ and $\mathrm{PETO}_{2}$ with no increase in $\mathrm{VE} \cdot \mathrm{VCO}_{2}{ }^{-1}$. The VT2 was determined by an increase in both $\mathrm{VE} \cdot \mathrm{V} \cdot \mathrm{O} 2^{-1}$ and $\mathrm{VE} \cdot \mathrm{VCO}_{2}$ and a decrease in $\mathrm{PETCO}_{2}$. The maximal aerobic speed (MAS) was associated with the last completed 30-s stage before exhaustion (Esteve-Lanao, et al., 2007). On the second day, 72 hours after the first test, an assessment of muscle stiffness with TMG was performed first. Subsequently, the RE was evaluated for six minutes.

\section{TMG measurements}

Measurements were taken with TMG (Tensiomyography System 100, Ljubljana, Slovenia) on the back of the lower limbs and with the subject in a prone position. Subjects were supported in this position by using a standard foam cushion under the distal third of the tibia to keep the ankle in a relaxed position. Caution was taken to avoid supporting the ankle and foot with the foam cushion and/or couch. The following variables were measured: delayed time (Td), contraction time (Tc), maximal radial displacement of the muscle belly (Dm), contraction time (Tc), and half relaxation time (Tr). Self-adhesive electrodes (Model 3100C, Uni Patch, Wabasha, $\mathrm{MN}$ ), with a diameter of $3.2 \mathrm{~cm}$, were placed longitudinally on the SO, GM and GL muscles of both legs in accordance to previous studies (Delagi, Perotto, Iazetti, \& Morrison, 1975) that placed the cathode proximally to the anode. Using a tripod, a digital displacement transductor (GK 40, Panoptik d.o.o., Ljubljana, Slovenia) was placed perpendicular to the muscle belly deformation with an initial pressure of $0.135 \mathrm{MPa}$. To determine the initial pressure, the evaluator had a reference on the linear transducer, which was generated by calculating the pressure exerted by the spring at a certain stretch point. Finally, the TMG-S2 (EMF-FURLAN \& Co. d.o.o., Ljubljana, Slovenia; 0-110mA) stimulator gave an electrical current intensity of 100 $\mathrm{mA}$ during 1 millisecond $(0.5-2 \mathrm{~ms})$ for each point.

Table 1. Physical characteristics and performance of participants $(n=9)$

\begin{tabular}{|c|c|c|c|c|}
\hline & Economic $(n=5)$ & Non-economic $(n=4)$ & $\mathrm{p}$ & ES \\
\hline Age (years) & $39.2(10.0)$ & $42.0(12.7)$ & 0.905 & 0.23 \\
\hline Weight (kg) & $72.0(8.6)$ & $72.5(12.9)$ & 0.905 & 0.05 \\
\hline Height (cm) & $178.2(4.6)$ & $175.3(5.3)$ & 0.190 & 0.58 \\
\hline $\mathrm{BMI}\left(\mathrm{kg} \cdot \mathrm{m}^{-2}\right)$ & $22.7(2.2)$ & $23.5(3.2)$ & 0.730 & 0.29 \\
\hline Experience (years) & $6.80(3.6)$ & $6.30(2.1)$ & 0.556 & 0.17 \\
\hline VO2 peak $\left(\mathrm{mL} \cdot \mathrm{kg}^{-1} \cdot \mathrm{min}^{-1}\right)$ & $56.6(7.5)$ & $53.6(2.9)$ & 0.413 & 0.52 \\
\hline Time 10 km (min) & $38.7(8.5)$ & $44.0(1.9)$ & 0.413 & 0.86 \\
\hline $\mathrm{RE}\left(\mathrm{mL} \cdot \mathrm{kg}^{-1} \cdot \mathrm{km}^{-1}\right)$ & $186.9(13)$ & $235.6(14.0)$ & $0.016^{*}$ & 3.60 \\
\hline
\end{tabular}

Note. Values are mean (standard deviation). Abbreviations: BMI: body mass index; VO2 peak: oxygen uptake; RE: running economy. *Significant differences between economic vs non-economic groups at $p \leq .05$ 
Prior to this stimulation, a familiarization stimulation of $40 \mathrm{~mA}$ was performed, respecting $30 \mathrm{~s}$ of recovery between the stimuli. (Piqueras-Sanchiz, et al., 2020).

According to the recommendations of Rodríguez-Matoso et al. (2012), all TMG measurements were done under static and relaxed conditions by a specialized researcher with more than four years of tensiomyography experience.

\section{Running economy}

For the RE determination, runners completed a 6-minute constant load treadmill running test at a $1 \%$ inclination, before the maximal graded test during the first testing session. All subjects avoided hard training sessions three days before evaluations on an individual basis (Esteve-Lanao, et al., 2019). The first ventilatory threshold (VT1) was chosen as the relevant physiological intensity for the RE evaluation (Barnes \& Kilding, 2015) and the average VO2 for the last three minutes of the treadmill test was used to measure the oxygen cost of running $\left(\mathrm{mL} \cdot \mathrm{kg}^{-1} \mathrm{~km}^{-1}\right)$. Breath-by-breath $\dot{\mathrm{V} O} 2$ was measured and averaged every 30 seconds during the test using the gas analyzer. In addition, heart rate (HR) was measured with a heart rate monitor (Polar RC3 GPS, Polar Electro, Finland).

\section{Statistical analysis}

Mean and standard deviation were used to inform descriptive statistics. To perform inferen- tial analysis, the Mann-Whitney U-test was used to compare TMG mechanical properties between the economic and non-economic runners. Cohen's d (Cohen, 1992) was calculated to inform effect size, considering small (.20), medium (.50), and large effect (.80). The significance level was set at $\mathrm{p} \leq .05$. Statistical analysis was done with SPSS v.21 (IBM, Armonk, NY).

\section{Results}

Significant differences $(p \leq .05)$ were found between the economic and non-economic runners in soleus muscle in $\mathrm{Td}$ (both sides) and Tc and Dm (right side) and $\operatorname{Tr}$ (left side) (Table 2). The most economic athletes showed faster contraction times versus non-economic athletes that showed lower values in $\mathrm{Td}$ and $\mathrm{Tc}$. In addition, no statistical differences were reported in the left leg.

According to GM values, the data were similar comparing to SO. Significant differences were found in Tc in the right GM (Table 3). The most economic runners reported lower Tc values in comparison to the non-economic runners. Therefore, no statistical differences were obtained in the Td, Tc, Dn, and TR parameters.

Finally, GL (Table 4) presented fewer similarities with the SO and GM. No clear differences were observed in the contraction process ( $\mathrm{Td}$ and $\mathrm{Tc}$ ) or stiffness. However, significant differences were found in $\mathrm{Tr}$ and $\mathrm{Ts}$ in the left leg.

Table 2. Soleus TMG differences between the economic and non-economic runners

\begin{tabular}{|c|c|c|c|c|c|c|c|c|}
\hline & \multicolumn{4}{|c|}{ Right } & \multicolumn{4}{|c|}{ Left } \\
\hline & Economic & Non-economic & $\mathrm{p}$ & ES & Economic & Non-economic & $\mathrm{p}$ & ES \\
\hline $\mathrm{Td}(\mathrm{ms})$ & $17.3(2.2)$ & $21.3(0.5)$ & $0.014^{*}$ & 2.36 & $18.9(1.6)$ & $22.2(1.9)$ & $0.027^{*}$ & 1.90 \\
\hline Tc (ms) & $32.4(22.5)$ & $58.2(6.9)$ & $0.050^{*}$ & 1.47 & $33.4(19.3)$ & $51.4(15.5)$ & 0.142 & 1.01 \\
\hline $\mathrm{Dm}(\mathrm{mm})$ & $2.9(2.7)$ & $7.5(0.9)$ & $0.050^{*}$ & 2.17 & $2.7(2.5)$ & $5.8(1.5)$ & 0.086 & 1.46 \\
\hline $\operatorname{Tr}(\mathrm{ms})$ & $88.9(57.4)$ & $104.6(55.6)$ & 0.462 & 0.28 & $53.2(21.3)$ & $105.2(42.7)$ & 0.050 & 1.61 \\
\hline Ts (ms) & $225.4(72.7)$ & $209.8(51.6)$ & 0.624 & 0.24 & $186.4(30.7)$ & $238.2(59.5)$ & 0.142 & 1.14 \\
\hline
\end{tabular}

Note. Values are mean (standard deviation). Abbreviations: $\mathrm{Td}$ - delay time; Tc - contraction time; Dm - maximal deformation; $\mathrm{Tr}$ relaxation time; Ts - sustentation time. * Significant differences at $p \leq .05$.

Table 3. Gastrocnemius medialis TMG differences between the economic and non-economic runners

\begin{tabular}{|c|c|c|c|c|c|c|c|c|}
\hline & \multicolumn{4}{|c|}{ Right } & \multicolumn{4}{|c|}{ Left } \\
\hline & Economic & Non-economic & $\mathrm{p}$ & ES & Economic & Non-economic & $p$ & ES \\
\hline $\mathrm{Td}(\mathrm{ms})$ & $20.8(1.8)$ & $23.1(0.7)$ & 0.086 & 1.60 & $22.1(2.3)$ & $24.5(1.5)$ & 0.142 & 1.20 \\
\hline $\mathrm{Tc}(\mathrm{ms})$ & $23.2(3.2)$ & $37.5(16.2)$ & $0.027^{*}$ & 1.31 & $28.1(4.7)$ & $39.7(17.7)$ & 0.960 & 0.96 \\
\hline $\mathrm{Dm}(\mathrm{mm})$ & $5.9(1.9)$ & $7.6(2)$ & 0.142 & 0.87 & $5.8(2.9)$ & $8.2(0.6)$ & 0.108 & 1.08 \\
\hline $\operatorname{Tr}(\mathrm{ms})$ & $50.5(48.9)$ & $57.3(14.6)$ & 0.142 & 0.18 & $58.2(47.9)$ & $64.9(19.5)$ & 0.170 & 0.17 \\
\hline Ts (ms) & $230.5(73.5)$ & $231(62)$ & 1.000 & 0.01 & $200.2(45.1)$ & $230.9(50.1)$ & 0.650 & 0.65 \\
\hline
\end{tabular}

Note. Values are mean (standard deviation). Abbreviations: Td - delay time; Tc - contraction time; Dm - maximal deformation; $\mathrm{Tr}$ relaxation time; Ts - sustentation time. * Significant differences at $p \leq .05$. 
Table 4. Gastrocnemius lateralis TMG differences between the economic and non-economic runners

\begin{tabular}{|c|c|c|c|c|c|c|c|c|}
\hline & \multicolumn{4}{|c|}{ Right } & \multicolumn{4}{|c|}{ Left } \\
\hline & Economic & Non-economic & $p$ & ES & Economic & Non-economic & $\mathrm{p}$ & ES \\
\hline $\mathrm{Td}(\mathrm{ms})$ & $19.8(1.5)$ & $19.8(0.9)$ & 0.624 & 0.00 & $20.2(2.7)$ & $21.5(0.7)$ & 0.624 & 0.62 \\
\hline $\mathrm{Tc}(\mathrm{ms})$ & $35.1(11.6)$ & $25.2(2.2)$ & 0.221 & 1.11 & $27.2(9.8)$ & $27.8(4.4)$ & 0.462 & 0.08 \\
\hline $\mathrm{Dm}(\mathrm{mm})$ & $6.3(2)$ & $6.7(1.4)$ & 1.000 & 0.23 & $5.1(1.6)$ & $7.2(0.9)$ & 0.086 & 1.56 \\
\hline $\operatorname{Tr}(\mathrm{ms})$ & $67.6(49.8)$ & $71.2(32.1)$ & 0.221 & 0.08 & $40.5(13.1)$ & $59.9(16.2)$ & $0.050^{*}$ & 1.34 \\
\hline Ts (ms) & $200(47)$ & $262(40.4)$ & 0.086 & 1.40 & $177.3(25.3)$ & $239.8(29.8)$ & $0.050^{*}$ & 2.29 \\
\hline
\end{tabular}

Note. Values are mean (standard deviation). Abbreviations: Td - delay time; Tc - contraction time; Dm - maximal deformation; $\mathrm{Tr}$ relaxation time; Ts - sustentation time. *Significant differences at $p \leq .05$.

\section{Discussion and conclusions}

Running economy can be evaluated at different physiological intensities. In our study, we selected VT1 to estimate RE in order to favor a more stable behavior of VO2 (Hamard, Laffite, Demarle, Koralsztein, \& Billat , 2000) since the slow component of $\mathrm{VO} 2$ was more pronounced at physiological intensities above VT1. To our knowledge, this is the first study that has evaluated the mechanical characteristics of muscle in runners in conjunction with RE. Significant differences $(p \leq .05)$ were found in the mechanical characteristics of the triceps surae musculature between the economic and non-economic runners at VT1. These differences were observed in parameters related to the response times ( $\mathrm{Td}$ and $\mathrm{Tc})$, muscle relaxation $(\mathrm{Tr})$, and level of muscular stiffness (Dm).

According to the parameters that provide information on muscle response time, the $\mathrm{Td}$ was, on average, between 15 to $19 \%$ lower in the SO in the group of economic runners. In addition, the Tc was lower between 35 to $44 \%$ in the SO and 29 to $38 \%$ in the GM in the more economic ones. In addition, Tr was lower in GL and SO in the more economic runners. It has been shown that the values of Td, Tc, and $\operatorname{Tr}$ depend on the type of predominant muscle fibers (Dahmane, Djordjevic, Simunic, \& Valencic, 2005). Simunič et al. (2011) showed a high correlation between the proportion of myosin heavy chain 1 and Td, Tc, and Tr on the vastus lateralis (VL). Despite being produced in a different muscle, our results show that the most economic group of runners have lower response times, which may be justified by lower support times in these runners. In addition, a rapid pre-activation that occurs during the flight phase has been described (Nummela, et al., 2007; Saunders, et al., 2004). This ability would allow runners to reduce the time necessary to achieve muscle activation during the support phase, improving RE (Tartaruga, et al., 2012). In spite of the fact that with the parameters measured by the TMG it is not possible to describe the moment in which activation starts, lower values in $\mathrm{Td}$ and $\mathrm{Tc}$ could help to this rapid activation. This pre-activa- tion process enhances alpha-gamma coactivation potentiating stretch reflexes and enhancing muscletendon stiffness (Kyröläinen, Belli, \& Komi, 2001; Saunders, et al., 2004), allowing better transmission of forces and less energy loss. These adaptations can be produced by a long-term exposure to specific load patterns such as training volume (Wiesinger, Hieder, Kosters, Muller, \& Seynnes 2017).

The lower Tr observed in the GL and SO of the most economic runners could reduce the necessary repolarization time of the muscular membrane in these muscles, favoring a new cycle of muscular contraction (Belic, Knez, Karba, \& Valenčič, 2000). In addition, lower values of $\operatorname{Tr}$ are associated with a situation of minor fatigue (Belic, et al., 2000). These neuromuscular benefits affect the voluntary and reflex neural activation, the mechanics of the race, and RE (Dumke, et al., 2010; Millet, et al., 2002). They also play a fundamental role in muscle mechanics (Dumke, et al., 2010; Millet, et al., 2002).

Muscular mechanics (Dm) allows to assess the maximal radial displacement of the muscle belly of the muscular group (Rodríguez-Matoso, et al., 2012). Lower Dm values represent high stiffness or muscle tone and muscular rigidity. In contrast, higher Dm values imply a lack of muscle tone or low stiffness. In our study, SO presented low Dm in the economic runners, which would improve the process of transmission of forces through the muscle, tendon, and ligament, minimizing the loss of elastic energy (Saunders, et al., 2004). This would explain the positive relationship between leg stiffness and RE (Dumke, et al., 2010; Spurrs, et al., 2003). This finding is consistent with previous studies, suggesting that higher-level athletes present greater stiffness in the running cycle when compared to lower-level athletes (Arampatzis, et al., 2006; Dumke, et al., 2010; Fletcher, et al., 2010, Spurrs, et al., 2003). The training process allows runners to have a positive adaptation in their mechanical architecture, increasing stiffness. Thus, athletes who are more economic present higher pennation angles (Abe, Kumagai, \& Brechue2000) and connective tissues (tendons, fascia, ...) that are capable 
of supplying and releasing elastic energy during muscle contraction to amplify energy production during the support phase (Wiesinger, et al., 2017). These adaptations allow runners to reduce hysteresis, thus contributing to a better transmission of forces with a lower energy cost.

During running, the central nervous system coordinates the action of muscles, ligaments, and tendons (Dumke, et al., 2010; Millet, et al., 2002). A reduced contact time during the support phase and better transmission of forces through greater stiffness could explain the combination of the neural (Td, Tc and Tr) and mechanical (Dm) differences that exist between the triceps surae of economic and non-economic athletes, consequently the group of economic runners could maximize kinetic benefits and RE (Santos-Concejero, et al., 2013, 2014; Saunders, et al., 2004).
In our study, the highest RE is also reflected in the time achieved in a $10-\mathrm{km}$ competition: the most economic group of runners took 5 minutes and 18 seconds less to complete the test. This is consistent with the studies showing that higher RE is related to better performance (Santos-Concejero, et al., 2014; Saunders, et al., 2004). According to our results, the biggest differences between the mechanical characteristics of runners are observed in the OS and the GM, but not as much in the GL. This may be due to the fact that OS and GM are the main contributors of propulsion and support in the propulsion phase of running at submaximal efforts (Ball \& Scurr, 2011; Hamner, et al., 2010 ). The main finding of our study is that the most economic runners have better response characteristics and greater stiffness of the triceps surae musculature - aspects that seem to be associated with better performance in runners.

\section{References}

Abe, T., Kumagai, K., Brechue W.F. (2000). Fascicle length of leg muscles is greater in sprinters than distance runners. Medicine and Science in Sports and Exercise, 32(6):1125-9. doi: 10.1097/00005768-200006000-00014.

Albertus-Kajee, Y., Tucker, R., Derman, W., Lamberts, R.P., \& Lambert, M.I. (2011). Alternative methods of normalising EMG during running. Journal of Electromyography and Kinesiology, 21(4), 579-586. doi: 10.1016/j. jelekin.2010.07.011

Arampatzis, A., De Monte, G., Karamanidis, K., Morey-Klapsing., G., Stafilidis., S., \& Brüggemann, G.P. (2006). Influence of the muscle-tendon unit's mechanical and morphological properties on running economy. Journal of Experimental Biology, 209, 3345-3357. doi: 10.1242/jeb.02340

Ayala, F., Moreno-Pérez, V., Vera-Garcia, F.J., Moya, M., Sanz-Rivas, D., \& Fernandez-Fernandez, J. (2016). Acute and time-course effects of traditional and dynamic warm-up routines in young elite junior tennis players. Plos One, 11, e0152790. doi: 10.1371/journal.pone.0152790.

Ball, N., \& Scurr, J.C. (2011). Efficacy of current and novel electromyographic normalization methods for lower limb highspeed muscle actions. European Journal of Sport Science, 11(6), 447-456. doi: 10.1080/17461391.2010.536583,

Balsalobre-Fernández, C., Santos-Concejero, J., \& Grivas, G.V. (2016). Effects of strength training on running economy in highly trained runners: A systematic review with meta-analysis of controlled trials. Journal of Strength and Conditioning Research, 30(8), 2361-2368. doi: 10.1519/JSC.0000000000001316

Barnes, K.R., \& Kilding, A.E. (2015). Running economy: Measurement, norms, and determining factors. Sports Medicine Open, 1, Art. No. 8. doi: 10.1186/s40798-015-0007-y

Belic, A., Knez, N., Karba, R., \& Valenčič, V. (2000). Validation of the human muscle model. In Proceedings of the 2000 Summer Computer Simulation Conference, 16-20 July, 2000, Vancouver, British Columbia. Session 1: Issues on whole body modeling.

Bosco, C., Montanari, G., Tarkkai, I., Latteri, F., Cozzi, M., \& Iachelli, G. (1987). The effect of pre-stretch on mechanical efficiency of human skeletal muscle. Acta Physiologica Scandinavica, 131(3), 323-329. doi: 10.1111/j.17481716.1987.tb08246.x

Burtscher, M., Gatterer, H., Faulhaber, M., Gerstgrasser, W., \& Schenk, K. (2010). Effects of intermittent hypoxia on running economy. International Journal of Sports Medicine, 31, 644-650. doi: 10.1055/s-0030-1255067

Cohen, J.A. (1992). A power primer. Psychological Bulletin, 112(1), 155-159. doi: 10.1037//0033-2909.112.1.155

Dahmane, R., Djordjevic, S., Simunic, B., \& Valencic, V. (2005). Spatial fiber type distribution in normal human muscle. Histochemical and tensiomyographical evaluation. Journal of Biomechanics, 62(12), 2451-2459. doi: 10.1016/j.jbiomech.2004.10.020

Delagi, E.F., Perotto, A., Iazetti, J., \& Morrison, D. (2011). Anatomic guide for the electromyographer. Charles Thomas.

Dumke, C.L., Pfaffenroth, C.M., McBride, J.M., \& McCauley, G.O. (2010). Relationship between muscle strength, power and stiffness and running economy in trained male runners. International Journal of Sports Physiology and Performance, 5(2), 249-261. doi: 10.1123/ijspp.5.2.249 
Esteve-Lanao, J., Del Rosso, S., Larumbe-Zabala, E., Cardona, C., Alcocer-Gamboa, A., Boullosa, D.A. (2019). Predicting recreational runners' marathon performance time during their training preparation. Journal of Strength and Conditioning Research, 2019 (In press) doi: 10.1519/JSC.0000000000003199

Esteve-Lanao, J., Foster, C., Seiler, S., \& Lucia, A. (2007). Impact of training intensity distribution on performance in endurance athletes. Journal of Strength and Conditioning Research, 21(3), 943-949. doi: 10.1519/R-19725.1

Fletcher, J.R., Esau, S.P., \& MacIntosh, B.R. (2010). Changes in tendon stiffness and running economy in highly trained distance runners. European Journal of Applied Physiology, 110(5), 1037-1046. doi: 10.1007/s00421-010-1582-8

Green, H.J., Roy, B., Grant, S., Hughson, R., Burnett, M., Otto, C.,..., \& Johnson, M. (2000). Increases in submaximal cycling efficiency mediated by altitude acclimatization. Journal of Applied Physiology, 89(3), 1189-1197. doi: 10.1152/jappl.2000.89.3.1189

Hamard, L., Laffite, L.S., Demarle, A., Koralsztein, J.P., \& Billat, V. (2000). Oxygen-pulse steady state allows estimation of $\dot{\mathrm{V} O} 2$ by heart rate monitoring in a severe submaximal run. Biology of Sports, 17(4), 255-263.

Hamner, S.R., Seth, A., \& Delp, S.L. (2010). Muscle contributions to propulsion and support during running. Journal of Biomechanics, 43(14), 2709-2716. doi: 10.1016/j.jbiomech.2010.06.025

Jones, A.M., \& Doust, J.H. (1996). A 1\% treadmill grade most accurately reflects the energetic cost of outdoor running. Journal of Sports Sciences, 14(4), 321-327. doi: 10.1080/02640419608727717

Kubo, K., Kanehisa, H., Ito, M., \& Fukunaga, T. (2001). Effects of isometric training on the elasticity of human tendon structures in vivo. Journal of Applied Physiology, 91(1), 26-32. doi: 10.1152/jappl.2001.91.1.26

Kyröläinen, H., Belli, A., \& Komi, P.V. (2001). Biomechanical factors affecting running economy. Medicine and Science in Sports and Exercise, 33(8), 1330-1337. doi: 10.1097/00005768-200108000-00014

López-Samanes, Á., Moreno-Pérez, D., Maté-Muñoz, J.L., Domínguez, R., Pallarés, J.G., Mora-Rodriguez, R., \& Ortega, J.F. (2017). Circadian rhythm effect on physical tennis performance in trained male players. Journal of Sports Sciences, 35(21), 2121-2128. doi: 10.1080/02640414.2016.1258481

Lucia, A., Esteve-Lanao, J., Oliván, J., Gómez-Gallego, F., San Juan, A.F., Santiago, C., ..., \& Foster, C. (2006). Physiological characteristics of the best Eritrean runners - exceptional running economy. Applied Physiology, Nutrition, and Metabolism, 31(5), 530-540. doi: 10.1139/h06-029

Maeda, N., Urabe, Y., Tsutsumi, S., Fujishita, H., Numano, S., Takeuchi, T., ..., \& Kimura, H. (2018). Symmetry tensiomyographic neuromuscular response after chronic anterior cruciate ligament (ACL) reconstruction. Knee Surgery, Sports Traumatology, Arthroscopy, 26(2), 411-417. doi: 10.1007/s00167-017-4460-7

Millet, G.P., Jaouen, B., Borrani, F., \& Candau, R. (2002). Effects of concurrent endurance and strength training on running economy and VO(2) kinetics. Medicine and Science in Sports and Exercise, 34(8), 1351-1359. doi: 10.1097/00005768-200208000-00018

Myers, R. (2011). Anatomy trains: Myofascial meridians for manual and movement therapists. Elsevier.

Morin, J.B., Dalleau, G., Kyröläinen, H., Jeannin, T., \& Belli, A. (2005). A simple method for measuring stiffness during running. Journal Applied Biomechanics, 21(2), 167-180. doi: 10.1123/jab.21.2.167

Morin, J.B., Samozino, P., Zameziati, K., \& Belli, A. (2007). Effects of altered stride frequency and contact time on leg-spring behavior in human running. Journal of Biomechanics, 40(15), 3341-3348. doi: 10.1016/j. jbiomech.2007.05.001

Noakes, T.D. (2002). Lore of running (3rd ed.). Champaign, IL: Human Kinetics Publishers.

Novacheck, T.F. (1998). The biomechanics of running. Gait and Posture, 7(1), 77-95. doi: 10.1016/s0966-6362(97)00038-6

Nummela, A., Keränen, T., \& Mikkelsson, L.O. (2007). Factors related to top running speed and economy. International Journal of Sports Medicine, 28(8), 655-661. doi: 10.1055/s-2007-964896

Orizio, C. (2002). Comments on the letter “Accelerometer and mechanomyogram”. Journal of Biomechanics, $35,385$.

Piqueras-Sanchiz, F., Martín-Rodríguez, S., Pareja-Blanco, F., Baraja-Vegas, L., Blázquez-Fernández, J., Bautista, I.J., \& García-García, O. (2020). Mechanomyographic Measures of muscle contractile properties are influenced by electrode size and stimulation pulse duration. Scientific Reports, 10(1), 8192. doi: 10.1038/s41598-020-65111-Z

Rodríguez-Matoso, D., García-Manso, J.M., Sarmiento, S., de Saa, Y., Vaamonde, D., Rodríguez-Ruiz, D., ..., \& Da Silva, M. (2012). Evaluación de la respuesta muscular como herramienta de control en el campo de la actividad física, la salud y el deporte. [TITLE IN ENGLISH, PLEASE. In Spanish.] Revista Andaluza de Medicina del Deporte, 5(1), 28-40. doi: 10.1016/S1888-7546(12)70006-0

Santos-Concejero, J., Granados, C., Irazusta, J., Bidaurrazaga-Letona, I., Zabala-Lili, J., Tam, N., \& Gil, N. (2013). Differences in ground contact time explain the less efficient running economy in North African runners. Biology of Sport, 30(3), 181-187. doi: 10.5604/20831862.1059170

Santos-Concejero, J., Tam, N., Granados, C., Irazusta, J., Bidaurrazaga-Letona, I., Zabala-Lili, J., Gil, S.M. (2014). Stride angle as a novel indicator of running economy in well-trained runners. Journal of Strength and Conditioning Research, 28(7), 1889-1895. doi: 10.1519/JSC.0000000000000325

Saunders, P.U., Pyne, D.B., Telford, R.D., \& Hawley, J.A. (2004). Factors affecting running economy in trained distance runners. Sports Medicine, 34(7), 465-485. doi:10.2165/00007256-200434070-00005

Sawyer, B.J., Blessinger, J.R., Irving, B.A., Weltman, A., Patrie, J.T., \& Gaesser, G.A. (2010). Walking and running economy: Inverse association with peak oxygen uptake. Medicine and Science in Sports and Exercise, 42(11), 2122-2127. doi: 10.1249/MSS.0b013e3181de2da7 
Simunič, B., Degens, H., Rittweger, J., Narici, M., Mekjavić, I.B., \& Pišot, R. (2011). Noninvasive estimation of myosin heavy chain composition in human skeletal muscle. Medicine and Science in Sports and Exercise, 43(9), 16191625. doi: 10.1249/MSS.0b013e31821522d0

Simunič, B. (2019). Two-dimensional spatial error distribution of key tensiomyographic parameters. Journal of Biomechanics, 92, 92-97 doi: 10.1016/j.jbiomech.2019.05.035.

Smith, D.J.A. (2003). Framework for understanding the training process leading to elite performance. Sports Medicine, 33(15), 1103-1126. doi: 10.2165/00007256-200333150-00003

Spurrs, R.W., Murphy, A.J., \& Watsford, M.L. (2003). The effect of plyometric training on distance running performance. European Journal of Applied Physiology, 89(1), 1-7. doi: 10.1007/s00421-002-0741-y

Støren, Ø., Helgerud, J., \& Hoff, J. (2011). Running stride peak forces inversely determine running economy in elite runners. Journal of Strength and Conditioning Research, 25(1), 117-123. doi: 10.1519/JSC.0b013e3181b62c8a

Tartaruga, M.P., Brisswalter, J., Peyré-Tartaruga., L.A., Avila, A.O.V., Alberton, C.L., Coertjens, M., \& MartinsKruel, L.F. (2012). The relationship between running economy and biomechanical variables in distance runners. Research Quarterly for Exercise and Sports, 83(3), 367-375. doi: 10.1080/02701367.2012.10599870

Valenčič, V. (1990). Direct measurement of the skeletal muscle tonus. Advances in External Control of Human Extremities, 10, 575-584.

Valenčič, V., \& Knez, N. (1997). Measuring of skeletal muscles’ dynamic properties. Artificial Organs, 21(3), $240-242$. doi: 10.1111/j.1525-1594.1997.tb04658.x

Valenčič, V., Knez, N., \& Simunič, B. (2001). Tensiomyography: Detection of skeletal muscle response by means of radial muscle belly displacement. Biomedical Engineering, 1, 1-10.

Wiesinger, H.P., Rieder, F., Kosters, A., Muller, E., \& Seynnes, O.R. (2017). Sport-specific capacity to use elastic energy in the patellar and achilles tendons of elite athletes. Frontiers in. Physiology, 13(8), 132. doi: 10.3389/ fphys.2017.00132

Zubac, D. \& Šimunič, B. (2017). Skeletal muscle contraction time and tone decrease after 8 weeks of plyometric training. Journal of Strength and Conditioning Research, 31(6), 1610-1619. doi: 10.1519/JSC.0000000000001626

Submitted: April 9, 2019

Accepted: October 15, 2020

Published Online First: December 11, 2020

Correspondence to:

Álvaro López-Samanes, Ph.D.

Universidad Francisco de Vitoria

Madrid, Spain

E-mail: alvaro.lopez@ufv.es

\section{Acknowledgement}

We would like to thank the "Run Faster \& Better runners training group" for their participation in this project. 